\title{
A practitioner-driven research agenda for syndromic surveillance
}

\author{
Richard Hopkins ${ }^{2}$, Julia Gunn ${ }^{3}$, John Berezowski ${ }^{4}$ and Howard Burkom¹ \\ 1Johns Hopkins Applied Physics Laboratory, Laurel, MD, USA; ${ }^{2}$ University of Florida, Tallahassee, FL, USA; ${ }^{3}$ Department of \\ Communicable Disease Control, Boston Public Health Commission, Boston, MA, USA; ${ }^{2}$ Veterinary Public Health Institute, University \\ of Bern, Switzerland, Bern, Switzerland
}

\section{Objective}

To obtain feedback and seek future directions for an ISDS initiative to establish and update research questions in Informatics, Analytics, Communications, and Systems Research with the greatest perceived impact for improving surveillance practice.

\section{Introduction}

Over the past fifteen years, syndromic surveillance (SyS) has evolved from a set of ad hoc methods used mostly in post-disaster settings, then expanded with broad support and development because of bioterrorism concerns, and subsequently evolved to a mature technology that runs continuously to detect and monitor a wide range of health issues. Continued enhancements needed to meet the challenges of novel health threats with increasingly complex information sources will require technical advances focused on day-to-day public health needs.

Since its formation in 2005, the International Society for Disease Surveillance (ISDS) has sought to clarify and coordinate global priorities in surveillance research. As part of a practitioner-driven initiative to identify current research priorities in SyS, ISDS polled its members about capabilities needed by SyS practitioners that could be improved as a result of research efforts. A taskforce of the ISDS Research Committee, consisting of national and global subject matter experts (SMEs) in SyS and ISDS professional staff, carried out the project. This panel will discuss the results and the preferred means to determine and communicate priorities in the future.

Identified Practice-Oriented Priority Areas for Surveillance Research

\begin{tabular}{|c|}
\hline Analytics \\
\hline 1. Methods and systems to support the fusion of various types of data \\
\hline 2. Methods to adapt SyS approaches and systems to changing needs \\
\hline 3. Enhanced and adaptive detection algorithms \\
\hline 4. Methods and processes for monitoring and addressing data quality issues \\
\hline 5. Processes to develop and assess syndrome definitions \\
6. Predictive analytic models using surveillance data \\
\hline Informatics \\
\hline 7. Methods to process, categorize, and code unstructured data in electronic medical records \\
\hline 8. Methods to assess the added value of new data sources and data elements \\
Systems Research \\
\hline 9. Standardized methods to validate and evaluate syndromic surveillance systems \\
\hline 10. Decision support methods for public health surveillance \\
\hline Communications \\
\hline 11. Communication methods, processes, and tools to inform decision-making \\
\hline 12. Effective strategies for building workforce competency in SyS practice \\
\hline
\end{tabular}

\section{Keywords}

surveillance research; priorities; public health practice

\section{*Howard Burkom}

E-mail: howard.burkom@jhuapl.edu 